\title{
Development of a Method for Choosing Cloud Computing on the Platform of Paas for Servicing the State Agencies
}

\author{
Kapan Oralgazyolu Shakerkhan \\ Department of Informatics \& Information Technologies, Shakarim State University of Semey city, 071409, Qazaqstan, \\ Email: kapan-shakerkhan@mail.ru \\ Ermek Tolegenovich Abilmazhinov \\ Department of Informatics \& Information Technologies, Shakarim State University of Semey city, 071412, Qazaqstan, \\ Email: eras71@mail.ru
}

Received: 16 May 2019; Accepted: 23 July 2019; Published: 08 September 2019

\begin{abstract}
In the scientific work is presented the development of a method of selecting cloud computing platforms for servicing government agencies, which in future will form the Digital Government according to the Message of the President of the Republic of Qazaqstan N. Nazarbayev to the people of Qazaqstan «Qazaqstan way 2050: Unified goal, common interests, common future». And also in the article are considered forecasts of growth and the development of cloud services in various countries, including Qazaqstan. Based on the results of research of company «Boston Consulting Group» (BCG), reflected in the article «Qazaqstan on the way to the digital economy», according to the level of digitalization the economy of Qazaqstan occupies the 50-th ranking of 85 governments and is in a group with emerging digital economy. The digital divide between the leading governments and the lagging countries is increasing from year to year; therefore, the article reflects one of the ways to develop the digitalization of Qazaqstan's economy. Descriptions and characteristics of various companies included in the international rating of Cloud100 are described as the most successful and competitive companies in the world on cloud technologies. All companies from this list are distributed as the top 20 companies for PaaS services. A criterion for selecting companies of cloud services is proposed, taking into account international and national ICT requirements. The web-portal (www.cloud.kz) was developed to assess the quality of cloud services from various companies of the world, which are working on cloud-based services. The analysis of the introduction of cloud services in Qazaqstan, and their advantages, and benefits for the public sector was made. For the cloud-based service of the module PaaS are selected the most optimal and suitable companies from the Cloud100 list.
\end{abstract}

Ccs Concepts-Cloud computing $\rightarrow$ Programmable systems; Redundancy; Cloud $\bullet$ Services $\rightarrow$ Platform as a Service (PaaS)
Index Terms - Cloud services, cloud 100, cloud platforms, Microsoft, Amazon, Google, cloud-based service PaaS, cloud computing, public cloud services, SaaS, IaaS, PaaS.

\section{INTRODUCTION}

The relevance of the scientific work lies in connection with the implementation of the government program «Digital Qazaqstan» for 2017-2020 and with the planned transition of state agencies to the new model of informatization, which includes the introduction of technologies on cloud computing, which in turn will lead to an increase the efficiency of public administration and reduce the cost of information and communication technologies in the public sector. Also in the scientific work are presented innovative solutions for transition the economy of Qazaqstan as a whole, to digitalization, by applying cloud services in all sectors of the economy of the RQ, which affects the message of the President of the Republic of Qazaqstan from 10.01.2018 to the people of Qazaqstan, where are considered issues of digitalization of the entire economy of Qazaqstan. In the research work are reflected the problems of servicing state agencies with cloud services and their solutions. To solve this problem, by us was conducted an analysis of cloud services and was offered a cloud computing service based on the PaaS model. The main criteria for selecting cloud technologies are proposed by us, for their introduction into the state agencies of Qazaqstan, which are not in Qazaqstan. Taking into account the main selection criteria and the differential method for assessing the quality of services, companies were selected in the form of a table, as the most suitable for servicing the state agencies of Qazaqstan, by module PaaS. A web-portal for evaluating the quality of cloud services on the PaaS module from various companies of the world was developed. 


\section{ANALYSIS OF EXISTING RESEARCH}

Currently, there are four main trends in the IT industry: cloud, user mobility, large data (bigdata), and social systems. We're considering perspectives of development cloud services, which in turn are divided into traditional models (see Fig.1):

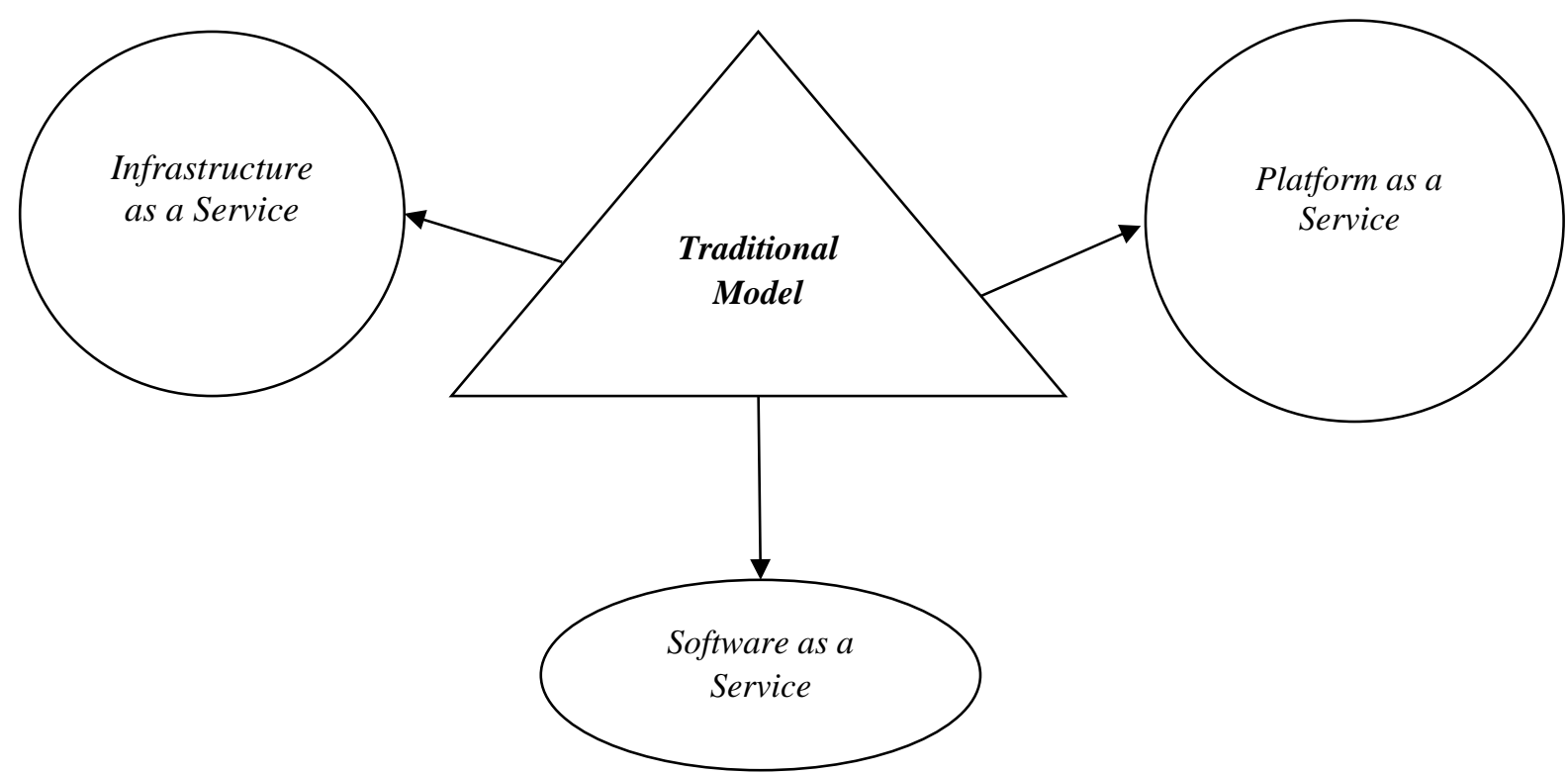

Fig.1. Traditional models of cloud services

And recently appeared models (see Fig.2):

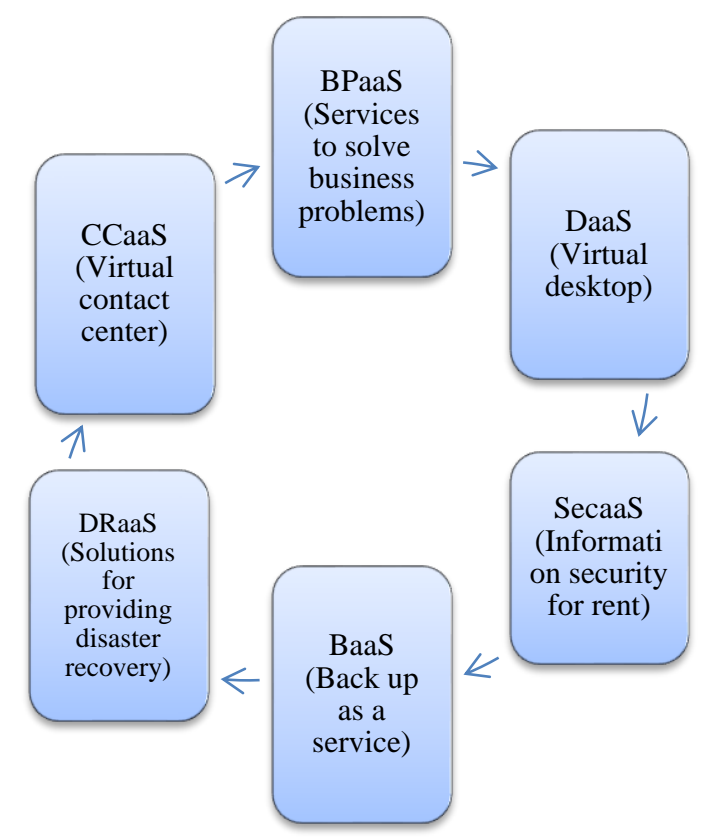

Fig.2. New models of cloud services

Fig. 3 presents, what part of the infrastructure remains in the company, and which is available as a service provider.

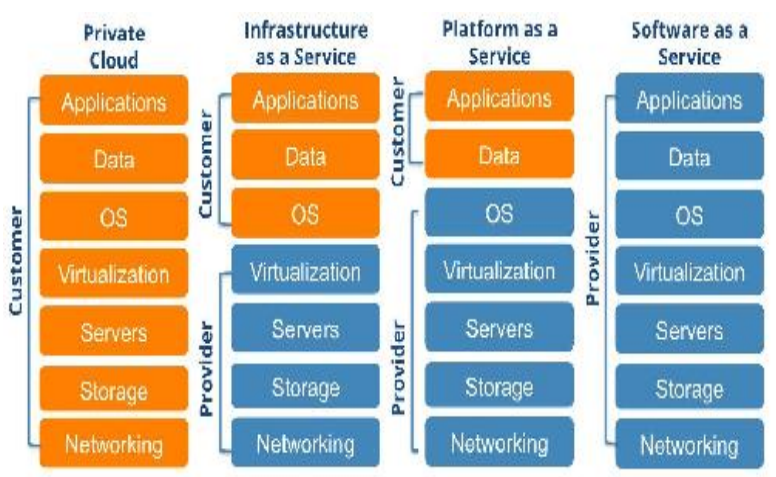

Fig.3. Models for providing cloud services

(source: http://www.jetinfo.ru/stati/iaas-paas-saas-razdel-territoriimezhdu-provajderami-i-klientami-oblachnykh-servisov)

The most advanced and technological market in this area is the US market. Studies show that in the United States the percentage of companies that use human resource management system (HRMS-Human Resources Management Systems), under the SaaS model is growing rapidly. The most known companies (service providers, outsourcing, business processes and IT) in this market have a long history of using SaaS business model. Their decisions are most in demand among companies with up to 1000 people, as well as among geographically dispersed or international companies that wish to consolidate business processes. 
According to market analysts, the HRM systems will be more focused on business requirements and less dependent on internal it-infrastructure of company. Analysts also note that the SaaS model is especially relevant in post-crisis conditions, when many companies highlight the importance of TCO/ROI (Total Cost of Ownership /Return on Investment) indicators, considering these metrics as «one of the determining factors in choosing HRM systems». According to IDC (analytical companies), SaaS market in the world amounted to about 20 billion dollars in 2011 and will grow to 60.8 billion dollars in 2016 year. However, according to STL (analytical company) information (see Fig.4), which brings its data on the secondary analysis basis of a number analytical agencies studying, including Bain Analysis, IDC, Forrester, William Blair \& Co., in 2017 the volume of this market may to reach 66 billion dollars.

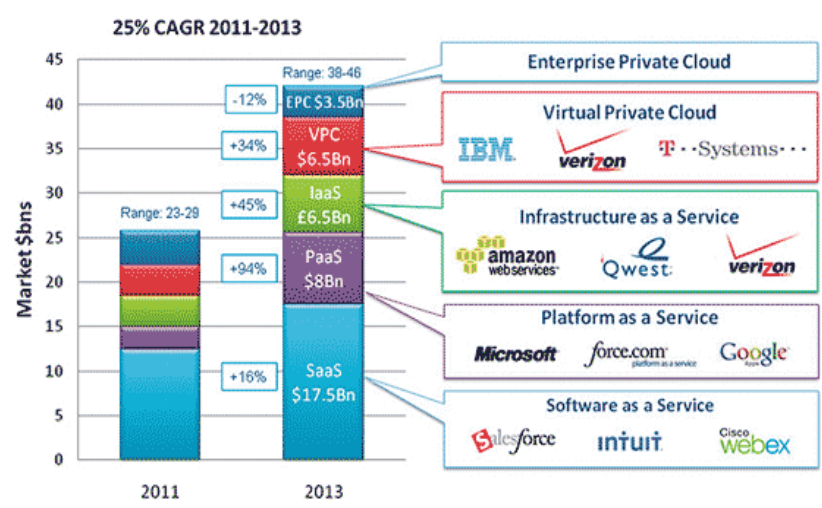

Fig.4. Estimates of the cloud computing market

(source: STL research-based Bain Analysis, Forrester, IDC, William Blair \& Co.)

What is a similar variation in the estimates?

Apparently, there are two groups of reasons.

The first - architectural and terminological - part of analysts adheres to the classical formulation, that is, defines SaaS as providing software in the form of a service based on a multi-tenant solution from the «cloud» for a wide range of unrelated clients, the presence of open API code, self-service elements and other classical attributes SaaS.

The second group of reasons for possible discrepancies is functionally terminological. For example, IDC as SaaS only considers traditional types of software, such as CRM, SCM, ERP, CAD / CAM / CAE, provided as services. Computer games, media services, e-shops, social networks and e-government services are classified by IDC as «business as a service», where services are built on the consumption of «cloud» IT services. [1,2,5]

With regard to the development of PaaS services, TechNavio analysts provided an industry report called «Global Platform as a Service (PaaS) for the period 20122016», which indicates that PaaS services market will reach $6.45 \$$ billion by 2016 . These numbers overlap with the forecasts of analytical companies Gartner, which said that the PaaS market will reach $\$ 1.2$ billion by the end of 2012. Despite the positive dynamics of growth in the popularity of PaaS, there are some difficulties, overcoming that the market can accelerate significantly above the forecasts of cloud technology experts. More than three quarters of the survey respondents said that they will have $41.5 \%$ of customers using only PaaS by 2016 , compared to $36.5 \%$ by the end of 2015 . More often clients of cloud model PaaS became: manufacture - $21.5 \%$ of respondents, and providing financial services - make $20.2 \%$ of respondents. Communications (16.1\%), government $(15.6 \%)$ and retail $(10.2 \%)$, finished the top five most popular clients of the cloud, presented a platform as a service. [4,6,8]

According to analysts, the world market of public cloud services (SaaS, IaaS, PaaS) will increase by an average of $40 \%$ annually and by 2016 the world market of public cloud services will reach $\$ 204$ billion, which is $16.5 \%$ more than in 2015 ( $\$ 175$ billion), predicts Gartner (see Fig.5). In turn, IDC expects even more growth - by $19.4 \%$ to $\$ 141$ billion against $\$ 70$ billion last year. According to Gartner forecasts by 2015, more than half of government services in the world will be provided from clouds, and many small and medium-sized businesses seriously consider the possibility of abandoning the traditional model of IT infrastructure in favor of public cloud services. According to foreign surveys, more than $60 \%$ of respondents are developing a strategy for the introduction of clouds, and a third is already actively exploiting them. Now the world's most popular services are private clouds - in a ratio of $60 / 40$, but by 2015 this proportion will be reversed. As a result, it turns out that modern cloud computing systems help to reduce the number of servers 6 times, the system takes up 6 times smaller area in the data center, besides it saves up to $80 \%$ of electricity. Gartner calculated that the world IT spending in 2016 will grow by $0.6 \%$ compared to last year. Thus, the growth rate of the public cloud services market is 27.5 times higher than the growth rate of the IT market as a whole. The IDC named a more modest number - the growth rates are six times different. The most growth, as Gartner expects, will be the «Infrastructure as a Service» segment (IaaS). According to analysts, in 2016 the growth of this direction in monetary terms will be $38.4 \%$. Cloud advertising - the largest segment of the global cloud services market, will show $13.6 \%$ growth in 2016 and reach $\$ 90.3$ billion while the volume of the IaaS segment is $\$ 22.4$ billion.

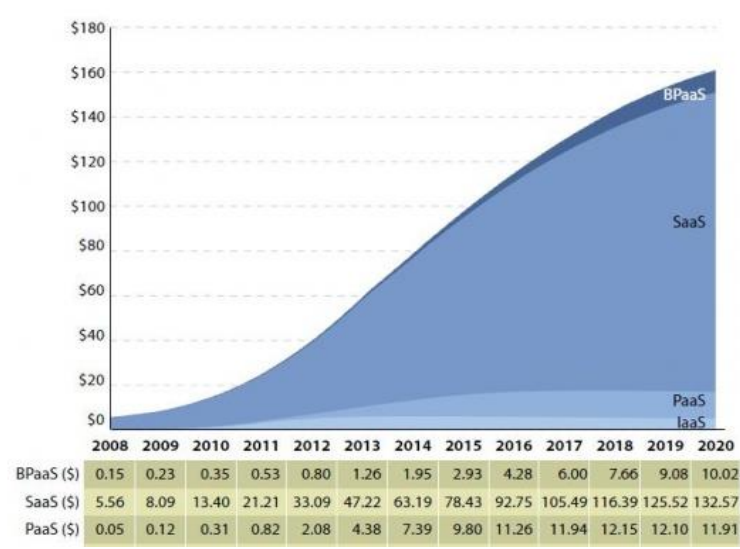

Fig.5 - the schedule of monitoring of cloud-based services (source: Forrester Research, 2015) 
Table 1. World expenses for public cloud services (billion \$)

\begin{tabular}{|c|c|c|c|c|}
\hline Type of service & $\mathbf{2 0 1 5}$ & Growth in 2015 & $\mathbf{2 0 1 6}$ & Growth in 2016 \\
\hline $\begin{array}{c}\text { Cloud business process services } \\
\text { (BPaS) }\end{array}$ & 39.2 & $2.7 \%$ & 42.6 & $8.7 \%$ \\
$\begin{array}{c}\text { Cloud application services } \\
\text { (SaaS) }\end{array}$ & 31.4 & $15.5 \%$ & 37.7 & $20.3 \%$ \\
$\begin{array}{c}\text { Cloud application infrastructure } \\
\text { services (PaaS) }\end{array}$ & 3.8 & $16.1 \%$ & 4.6 & $21.1 \%$ \\
$\begin{array}{c}\text { Cloud system infrastructure } \\
\text { services (IaaS) }\end{array}$ & 16.2 & $31.9 \%$ & 22.4 & $38.4 \%$ \\
\hline $\begin{array}{c}\text { Cloud management and security } \\
\text { services } \\
\text { Cloud advertising }\end{array}$ & 5.0 & $20.7 \%$ & 6.2 & $13.6 \%$ \\
\hline Total & 79.4 & $15.4 \%$ & 90.3 & $16.5 \%$ \\
\hline
\end{tabular}

The reasons for growth

«The high growth rate of the global market for cloud services providers are due to the transition from traditional patterns of service consumption to cloud services», explained Gartner analysts. Over the past few years the software industry has been actively proceeds to model development and implementation since the beginning of the cloud (SaaS). By 2018, most of the software vendors will go completely to the SaaS/PaaS code base. This means that many corporate customers, who have reached the next major stage of software update, will be first of all offered to use the SaaS model, IDC added.

\section{Vertical market}

Most public cloud services in 2015 y. were spent by piece production industry ( $\$ 8.6$ billion), further followed by banking services ( $\$ 6.8$ billion), and followed by professional services (\$ 6.6 billion). By 2019, professional services will overtake banking services in terms of spending on public clouds and will take second place. All three of these industries in 2015 were leaders in the EMEA (Europe, Middle East Africa). Telecommunications industry, in turn, will take the first place by 2019 in terms of expenses. In addition, the telecommunications industry for the period from 2014 to 2019 yr. will show the highest average annual growth rate at the level of $22.2 \%$. The value of more than $20 \%$ during the period also shows: media companies, federal and municipal government, education, retail, transportation and mining. [6,7,8,9]

Cloud development in different countries of the world.
The first opportunities for cloud computing were evaluated by American companies. Vivek Kundra director of the federal department of IT technologies CIO of the US government, in February 2011, published a strategy for the US government to transfer part of information systems to the cloud. The document entitled «Federal Cloud Computing Strategy» clearly describes the order, and the time to transfer the part of the system to the cloud. Their goal is to reduce the complexity of managing IT technologies, increase the utilization of equipment to $70-80 \%$, and reduce the number of data centers, now the US government has more than 800 . Currently, the United States Senate filed a new bill designed to adapt its legislation to the new realities of cloud computing. 2012 Cloud Computing Act, submitted to the Senate by the representatives of the Republican and Democratic Party, will oblige all federal agencies publish annual reports on the progress of transferring their computer networks to the cloud. Processing large massive of data sets is a very costly exercise because it requires serious computing power, respectively; need a lot of expensive servers. Nevertheless, in the US they found a way out of the situation, installed on a single hardware, several operating systems that allegedly work on separate computers. Due to this, the efficiency is increased to $85 \%$, if you compare these readings with each individual server, then their efficiency will be $10 \%$ per year. Therefore, $90 \%$ of time the system heats the air. In addition to increasing efficiency, there is considerable savings in technology, which simply does not need to be bought. In addition, such systems allow you to cope with scheduled peak loads. For example, we know that at the end of the month the accounting department will have a lot of work. To ensure that nothing «hangs» and does not «slow down», 
we allocate additional capacities on virtual servers. This process can occur in an automatic mode. And for each employee of the company you can create a virtual workplace. In the application of cloud technologies in Qazaqstan not so extensive experience is accumulated, nevertheless, there are enough examples of successful implementation. Among the government initiatives in the field of cloud computing, the strategy of the US government is the most extensive and ambitious. The administration of Barack Obama launched a radical ITreform, whose goal is to reduce costs, increase the transparency and the efficiency of government IT spending, and the federal cloud strategy is one of the most important tools for its implementation. [9,10,11,13]

The most prosperous country for «clouds» is Japan. Nowadays, it is there operating the most favorable, technological and legal framework, for effective implementation and development of cloud technologies. Japanese corporation Panasonic expands the line of «smart» household appliances and launches in Japan a new cloud service that allows to remotely controlling a whole range of home appliances, from refrigerators and air conditioners, to kitchen appliances, appliances for beauty and health. The Panasonic Smart App, which will be available from the end of September this year, will allow owners of Smartphone on the Android OS, remotely manage compatible appliances, setting various programs for devices and monitoring energy savings. This is not the first case of using cloud technology by Panasonic - in June 2012, the 3-Star Bistro microwave oven and induction rice cooker, owners who can choose recipes, and program settings, and upload them to kitchen appliances via Android Smartphones have already been introduced. On September 25, Panasonic will introduce in Japan new home appliances that support management from mobile devices. Among them are $\mathrm{X}$ series air conditioners, the flagship model of the refrigerator NRF557XV, the drum-type washing machines with built-in drying (NA-VX8200 / NA-VT8200), as well as the beauty and health equipment - the body parameters analyzer EW-FA43, calorie counter EW -NK63 and a tonometer EW-BW53. By the way, new models of air conditioners can be managed not only from Androidsmartphones, but also from mobile devices Apple iPhones. Using the Panasonic Smart App application, you can remotely control the operation of the home air conditioner, (while away from home), check the efficiency of the refrigerator or turn on the washing machine by setting the required washing program. The program will also help to maintain beauty and health, allowing users to efficiently manage body health data - to build graphs of body weight changes, to track the amount of calories consumed and energy spent on performing tasks. Thus, the development of cloud technology in Japan comes with advanced technologies. [2,11,13,17,18]

The experience of the Korean government, on the application of cloud computing technology, was noted as an excellent example in the UN report. The Economic Report for 2013, published by the United Nations Conference on Trade and Development (UNCTD) on
December 3, focused on the system, Korea's general national computing platform, describing it as a «standardized and automated system for managing a single government information center». The Economic Report 2013, published by UNCTD, stated that the national platform system improved the investment attractiveness of infrastructure, information and security systems. The report reported that effective management of public, information resources allowed reducing budget expenditures and increase energy efficiency, as well as opportunities for countering cyber-attacks. Two case studies, highlighted in the report, included a tremendous reduction in budget expenditures and a strengthened system of responses to cyber threats. Improved supply, shared resources, and coordinated development of common functions and services allowed to reduce budget expenditures for the purchase and operation of ICT resources in the region of $30 \%$. In addition, with the introduction of automated, information, recognition system that captures and blocks external attacks, the monthly average time loss radically decreased from 67 minutes per unit in 2004 to 3.04 seconds in June 2013. The report was characterized by the Korean government, as «the leading example of network compatibility and management of the data center». [11,20]

As for Russia, there is a vivid example of virtualization in it - it is a project of the mobile operator MTS and VMware. As a result of the introduction to virtualization technologies, power consumption and air conditioning costs were reduced by $20 \%$, saving of occupied physical space was $60 \%$, saving of used physical equipment $-78 \%$, cost saving for licensing (Windows Server licenses) 85.5\%. The total number of licenses decreased from 116 to 36. Developing its own cloud services that can be easily operated and added, sometimes it takes a few hours to fix the problem and launch some new service. As a result, it turns out that modern cloud computing systems help to reduce the number of servers 6 times, the system takes up to 6 times smaller area in the data center, besides it saves up to $80 \%$ of electricity. However, according to IDC research, the volume of the market of cloud IT services in Russia amounted to about \$ 35.08 million in 2010 , which is about $0.006 \%$ of the total IT market in Russia, whereas globally, the share of IT services for public clouds was for the same period about $7.5 \%$ of the world IT market. This is partly due to the fact that in Russia the share of services in the total volume of the IT market is about $20 \%$, whereas in the Western IT market, services account for more than $60 \%$. As the world experience shows, at the initial stage of the development of the cloud IT services market, the SaaS model dominates, which, as the market matures, lags behind the growing segments of IaaS and PaaS. In 2009, the Russian market was at the very beginning of development, and about $94 \%$ of its volume was accounted for by SaaS. The same trend continued in 2010. The main share in this segment, according to IDC, was Microsoft products, provided to customers through local partners of the company. The volume of the PaaS segment in 2009 in Russia was only slightly less than $5.5 \%$ of the cloud 
market, this market in Russia only then began to form. And in 2010 there were solutions based on the platform of the company Aprentis, then the Russian project was launched - the cloud platform Hivext, supported by Softline. This is the first in Russia portal, providing customers with a large selection of «cloud» solutions, and developers - a platform for development and a full cycle of promotion and sales of SaaS. However, with the existing offer from the largest international corporations Microsoft, IBM, Intel, NEC, as well as the Russian system integrator - companies CROC and other domestic IT-suppliers, the demand for cloud services in Russia is still small. Nevertheless, the IBM 2011 CIO Study showed that $60 \%$ of the organization is ready to implement solutions over the next five years based on cloud computing technology. Segment IaaS occupied in 2010 about $0.5 \%$ of the total market of cloud services. In 2009, «Oversan» and IT Grad announced the launch of the IaaS business in Russia. Later, they were added a number of players - primarily CROC and Parking.ru. According to the CROC, starting this business in 2010, in September the company signed a number of projects on cloud computing for a total of $\$ 800$ thousand. Finally, in 2010 , more than 200 companies and projects were located in the «Oversan» cloud. Countries with more developed markets for «cloud» services are following the same path: first saturation with SaaS services, and then the share of this segment is gradually reduced. For example, in 2008, the share of SaaS in the world market was $57 \%$, and in 2009 - about 50\%. According to IDC forecasts, as the cloud computing market matures, the share of Russian solutions on it will increase; while the market itself will grow at a high rate. By the end of 2014, according to IDC, the volume of the Russian SaaS market will increase to $\$ 113.4$ million, the IaaS segment will grow to $\$ 35.5$ million, and PaaS to $\$ 12.5$ million. Considering the development of cloud technologies in Russia, it should be noted that according to IDC analysts, the Russian cloud market is at an average stage of its development and the growing interest in the cloud model of providing IT services is noticeable. Indeed, Russia is a huge country with a moderately developing it-infrastructure; this alone is enough for the success of the cloud revolution in Russia Using the clouds, authorities, municipalities, as well as local companies could finally get access to high-quality modern and inexpensive IT-systems. [1,9, 15,16]

At 2011 year in Qazaqstan is launched the development of cloud projects to keep up, and be head and shoulders above other countries. The curator of cloud projects was the daughter company of Qazaqtelecom JSC»KT Cloud Lab». And in the metropolitan area of Qazaqstan, in Almaty the representative office of the company «NEC Neva Communication Systems» was opened. Representatives of these companies expressed their readiness to meet the needs of small and mediumsized businesses in Qazaqstan in IT solutions in the field of cloud computing. On the IT market of Qazaqstan, NEC has been operating since 2006. The main customer of this company is Qazaqtelecom. Other IT representatives of Qazaqstan, such as KazTransCom, also use NEC services.
Successfully developing in the field of cloud technologies, such companies as Academset LLP, ST Integrator Company LLP, ARTA ${ }^{\mathrm{TM}}$ Company, LLP KT-Cloud-Lab representatives from Cisco, Microsoft and many others. As for the demand for virtualization in business, the study «Study of demand for info communication services in the segment of corporate business» was conducted (ICTMarketing, at the end of 2012). According to its results, the Virtual Data Center service is attractive for $13 \%$ of companies in Almaty, $4 \%$ in Astana, as well as for $1 \%$ in Karaganda and $5 \%$ in Ust-Kamenogorsk, which amounts to 353 medium and large enterprises. Some Qazaq companies have gone further and have already built whole data centers for commercial operation and will launch them in the near future. As an example, we can mention the project of Qazaqtelecom - «Private cloud environment» on the basis of data processing centers for corporate clients. We can also consider that the development of intelligent technologies in Qazaqstan, for their deployment in the cloud environment, is progressing well. For example, in the Institute of Informatics and Management Problems in Qazaqstan, a system of intelligent computer vision based on 3D technology has been developed. It is designed to create artificial images that mimic human behavior. Software and hardware complex «Azamat 911» was named as a domestic «Robocop». It is equipped with ten cameras, and the software allows you to focus on people whose behavior will seem to the Azamat-911 complex to be suspicious. As for the construction and implementation of the Data Centers of huge capacity, large-scale, undeveloped, both in Western Europe and the United States, but mainly the development of data centers of small-medium capacity, for the banking sector and for small and medium-sized businesses in Qazaqstan. One of the examples of such centers is in Pavlodar, with a capacity of 300 server cabinets, which is inferior to foreign analogues, but still the largest in Qazaqstan. [10,18]

\section{MAIN ObJECTIVE OF THE ReSEARCH}

The purpose of scientific work is a solution the problem of choosing platforms for cloud computing government agencies. In the process of scientific work, the following studies have carried out:

The analysis of the introduction of cloud services into Qazaqstan, and their advantages, and benefits for the public sector.

List of companies in the form of a table, as the most competitive and the best in the world among cloud services, by the module of PaaS.

The main criteria for selection of cloud technologies are proposed, for their introduction into state agencies of Qazaqstan.

To assess the level of quality of cloud services, a differential method of quality assessment is applied.

The proposed list of companies, in the form of a table, from the list of Cloud100 are rated by all major criteria, as the most competitive and the best among all cloud companies in the world, by the module of PaaS. 
Taking into account the main selection criteria and the differential method for assessing the quality of services, companies were selected in the form of a table, the most suitable for the service of state bodies of Qazaqstan, by the module of PaaS.

- $\quad$ Designed the web Portal for quality assessment of cloud services from various companies around the world.

\section{RELATED WORKS}

Among the main players in the cloud computing market are: Google, Microsoft, Amazon.com. The main services are Windows Azure, Google Apps Engine, Amazon Web Services (see Fig.5)

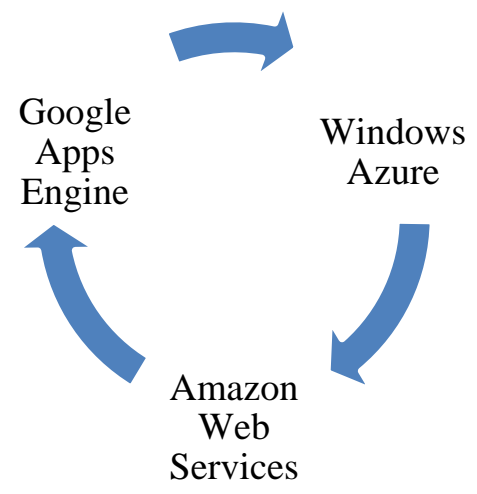

Fig.5 - Cloud services

It is proposed that a brief description software products of the major players involved in cloud-based services, such as Microsoft, Google.

Company Microsoft to provide cloud-based services uses its own product, called Windows Azure.

Windows Azure is an open and flexible cloud platform that allows you to quickly create, deploy, and manage applications in a global network of data centers running Microsoft. You can develop applications using any language, tool or platform. In addition, in some public cloud applications can integrate with existing IT environment.

From this definition it follows that Windows Azure is a cloud platform and allows you to work with business applications, services and tasks in the cloud.

Keywords describing the Windows Azure platform.

Flexible. Windows Azure platform includes many cloud services for any tasks - from website hosting companies to work with large databases of SQL in the cloud. In addition, this platform has features that provide high performance and low latency network for cloudbased applications.

Managed. All Windows Azure services are posted in several datacenters; in the United States, Europe and Asia. These centers are managed by Microsoft and provide round-the-clock, professional support.
Compatible. Cloud applications in Windows Azure can easily be integrated with local IT environments running on Microsoft Windows Server.

Windows Azure provides companies; four main types of cloud services: (see Fig.6)

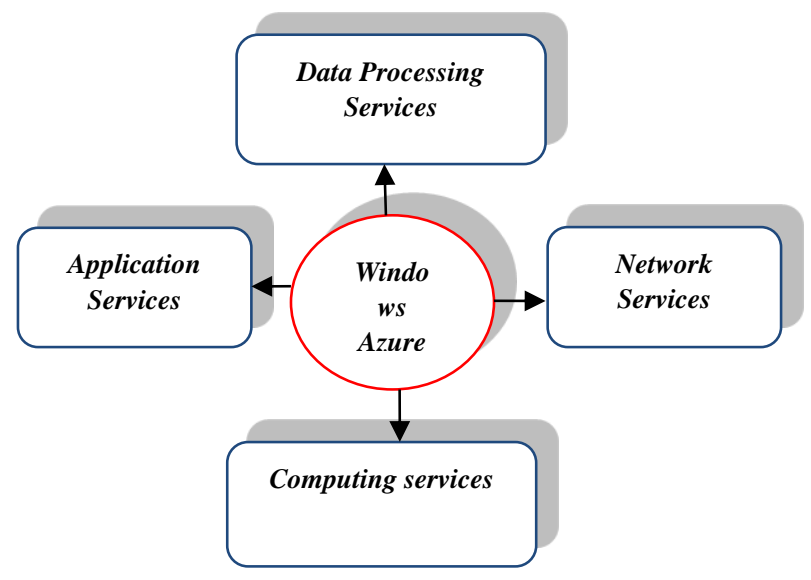

Fig.6 - Types of cloud services of Windows Azure

Google Company, to provide cloud-based services, uses its products Google Apps. Google App Engine allows you to run your web applications in the Google infrastructure. Applications of Google App Engine are easy to create, maintain and improve as traffic and data storage grows.

Google App Engine includes the following functions:

Dynamic work in the Internet with full support of basic Web technologies;

Persistent storage with queries, sorting and transactions;

Automatic scaling and load control;

APIs for authenticating users and sending email using Google accounts;

Full-featured local environment of development that simulates Google App Engine on your computer;

Scheduled tasks for track events at a specific time or at regular intervals. [7,8,17,19]

Basic criteria for selection cloud services (see Fig.7)

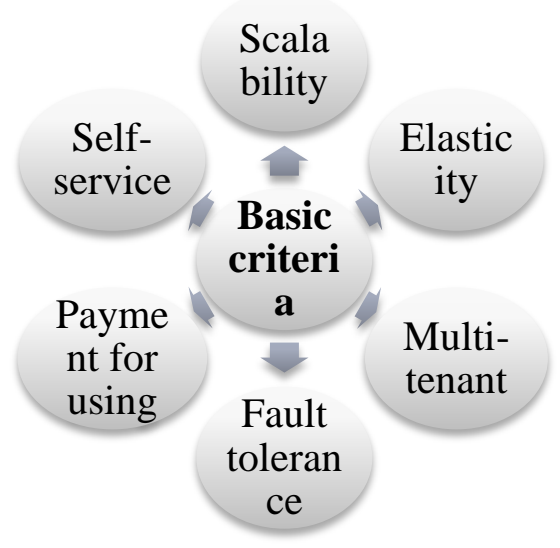

Fig.7 - The main criteria of selecting cloud services 
Scalability is the ability of information systems entities to withstand the growing workload and handle large amounts of data.

Elasticity - the ability to quickly respond to changing business conditions and the ability to rapidly introduce new products or services, having the complete cycle of planning, design and development information system.

Multi-tenancy is one of the ways to reduce costs by maximizing the using of common resources for servicing different groups of users, different organizations, different categories of consumers, and so on.

Payment of used resources is another attribute of cloud computing that allows you to transfer part of the capital costs to operating costs. Thus by purchasing only the required amount of resources, optimize the costs related to the work of the information systems organization.

Self-service is the ability to request and obtain the required resources to consumers in a matter of minutes. $[9,20]$

\section{Twenty leading providers of cloud platform.}

There are many ways to build cloud platforms. Consider the 20 most promising suppliers of the cloud platform from the Cloud 100 list that solve this problem (see Table 2).

Table 2 - The list of companies of PaaS module

\begin{tabular}{|c|c|c|c|c|c|}
\hline & \multicolumn{5}{|c|}{ Names of companies } \\
\hline $\mathbf{1}$ & Anypresence & AppFog & Apprenda & Cloudera & Cumulogic \\
\hline $\mathbf{2}$ & enStratus & GigaSpaces & Gizmox & Long jump & Mule Soft \\
\hline $\mathbf{3}$ & OS33 & Rightscale & Red Hat & Heroku & Parallels \\
\hline
\end{tabular}

To assess the level of quality of services using the following methods: differential, integral and mixed. In my work to assess the quality of cloud services, I chose a method such as differential evaluation method is more suitable for international standards for such services.

Differential method of assessing the level of quality is to compare single quality indicators evaluated services with relevant single base sample quality indicators (see formula 1). For each of the indicators are calculated relative indicators of quality:

$$
\mathrm{K}_{\mathrm{i}}=\frac{\mathrm{P}_{\mathrm{i}}}{\mathrm{P}_{\mathrm{i} \sigma}}
$$

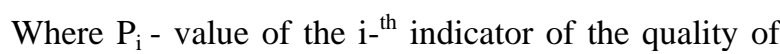
the evaluated service; $P_{i b}$ - value of the $i$-th index of the quality of the base sample. [2,12,19]

If the evaluated service has all relative quality indicators $\mathrm{Ki}>=1$, then its quality level is higher or equal to the base one; if all $\mathrm{Ki}<1$, then lower.

Cloud platforms are evaluated by such criteria as scalability, elasticity, multi-tenancy, fault tolerance, payment for using, self-service. Therefore, when assessing cloud services, for a basic sample, services are accepted whose quality indicators correspond to the world level or the best domestic samples. The selection of basic samples is carried out by the relevant industry research institutes, central and head design offices, parent and basic organizations for standardization. The level of quality of services, estimated by the aggregate of indicators, which does not include economic, is called the technical level of quality of services. When economic indicators are taken into account, then they speak about the technical and economic level of the quality of services. In my work, cloud services are mostly estimated by technical indicators. [1,9,19]

Consider a table list of companies (see Table 3), that evaluated on criteria such as scalability, elasticity, multitenancy, fault tolerance, payment for using, self service for technical reasons.

Brief description of company's services which are working in cloud-based PaaS services (see Table 4).

Table 3 - Criteria for evaluating PaaS services

\begin{tabular}{|c|c|c|c|c|c|c|c|}
\hline$\stackrel{\dot{0}}{\mathbf{z}}$ & 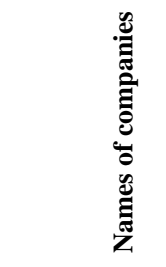 & 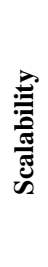 & 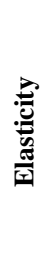 & 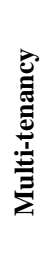 & 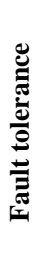 & 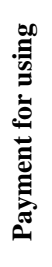 & 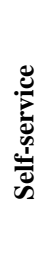 \\
\hline 1 & AnyPresence & 5 & 5 & 5 & 5 & 3 & 5 \\
\hline 2 & AppFog & 5 & 5 & 5 & 5 & 5 & 5 \\
\hline 3 & Apprenda & 5 & 5 & 5 & 5 & 4 & 5 \\
\hline 4 & CloudBees & 4 & 4 & 3 & 4 & 5 & 5 \\
\hline
\end{tabular}




\begin{tabular}{|c|l|c|c|c|c|c|c|}
\hline $\mathbf{5}$ & Cloudera & 5 & 5 & 5 & 5 & 3 & 5 \\
\hline $\mathbf{6}$ & Cumulogic & 5 & 5 & 5 & 5 & 3 & 5 \\
\hline $\mathbf{7}$ & Engine Yard & 4 & 4 & 4 & 3 & 4 & 5 \\
\hline $\mathbf{8}$ & enStratus & 5 & 5 & 5 & 5 & 4 & 5 \\
\hline $\mathbf{9}$ & GigaSpaces & 5 & 5 & 5 & 5 & 3 & 5 \\
\hline $\mathbf{1 0}$ & Gizmox & 5 & 5 & 5 & 5 & 5 & 5 \\
\hline $\mathbf{1 1}$ & Intalio & 4 & 4 & 4 & 4 & 3 & 4 \\
\hline $\mathbf{1 2}$ & LongJump & 5 & 5 & 5 & 5 & 5 & 5 \\
\hline $\mathbf{1 3}$ & Morphlabs & 4 & 4 & 4 & 4 & 4 & 4 \\
\hline $\mathbf{1 4}$ & MuleSoft & 5 & 5 & 5 & 5 & 4 & 5 \\
\hline $\mathbf{1 5}$ & OS33 & 5 & 5 & 5 & 5 & 3 & 5 \\
\hline $\mathbf{1 6}$ & Red Hat & 5 & 5 & 5 & 5 & 5 & 5 \\
\hline $\mathbf{1 7}$ & Rightscale & 5 & 5 & 5 & 5 & 5 & 5 \\
\hline $\mathbf{1 8}$ & Cloud Foundry & 4 & 4 & 4 & 3 & 4 & 4 \\
\hline $\mathbf{1 9}$ & Heroku & 5 & 5 & 5 & 5 & 5 & 5 \\
\hline $\mathbf{2 0}$ & Parallels & 5 & 5 & 5 & 5 & 5 & 5 \\
\hline
\end{tabular}

Table 4 - Description of cloud services PaaS

\begin{tabular}{|c|c|c|c|}
\hline No. $p / p$ & Names of companies & Advantages & Disadvantages \\
\hline 1 & AnyPresence & $\begin{array}{l}\text { Allows you to compile and deploy HTML5, } \\
\text { iOS and Android programs without installing } \\
\text { software. }\end{array}$ & Templates for mobile apps only \\
\hline 2 & AppFog & $\begin{array}{l}\text { Allows you to deploy applications in the } \\
\text { programming languages PHP, Ruby, Node.js } \\
\text { and Java. AppFog provides a reliable, scalable } \\
\text { and high-speed platform for applications in } \\
\text { the cloud Amazon, Rackspace, Microsoft, HP } \\
\text { and others. }\end{array}$ & None \\
\hline 3 & Apprenda & $\begin{array}{l}\text { Uses an open set of protocols PaaS for .Net } \\
\text { allows you to manage Windows applications } \\
\text { in any infrastructure. In addition, it is } \\
\text { compatible with Java. }\end{array}$ & $\begin{array}{l}\text { Prices are more expensive than } \\
\text { other companies }\end{array}$ \\
\hline 4 & CloudBees & $\begin{array}{l}\text { Allows you to quickly and easily build, } \\
\text { deploy, and manage Java applications in the } \\
\text { cloud. Provides services such as Build } \\
\text { services, Run services, Weave services. }\end{array}$ & $\begin{array}{l}\text { Not compatible with applications } \\
\text { developed in other programming } \\
\text { languages }\end{array}$ \\
\hline 5 & Cloudera & $\begin{array}{l}\text { The company launched a package of services } \\
\text { called Cloudera Enterprise, consisting of three } \\
\text { products: } \\
\text { CDH is the distribution of Apache Hadoop } \\
\text { (HDFS, MapReduce and MapReduce2, } \\
\text { Hadoop Common,, which includes a number } \\
\text { of related programs and libraries such as } \\
\text { Apache Flume, Apache Hive, Hue, Apache } \\
\text { Mahout, Apache Oozie, Apache Pig, Apache } \\
\text { Sqoop, Apache Whirr and Apache Zookeeper. } \\
\text { Cloudera Manager is a tool for deploying, } \\
\text { monitoring and managing an Apache Hadoop } \\
\text { cluster. } \\
\text { Cloudera Support is the professional support } \\
\text { provided by Cloudera specialists on issues } \\
\text { related to CDH and Cloudera Manager. }\end{array}$ & $\begin{array}{l}\text { Uses an improper, commercial } \\
\text { version of the software framework } \\
\text { Apache Hadoop. }\end{array}$ \\
\hline
\end{tabular}




\section{CONCLUSIONS}

We have designed web - portal (www.cloud.kz), to evaluate the quality of cloud services from various companies in the world that are working on cloud services. The web - portal is a platform where services are displayed for a segment such as PaaS. And the service in the $1^{\text {st }}$ segment is rated by all criteria, in the $5^{\text {th }}$ point scale. The quality of cloud services is estimated of using the differential methods and results are given in the form of a histogram. The web-portal provides the room for the registration of companies engaged in cloud services (see Fig.8).

For example, the results of evaluating the quality of cloud services by criteria such as a scalability and fault tolerance in the PaaS segment which have presented. (see Fig.9,10)

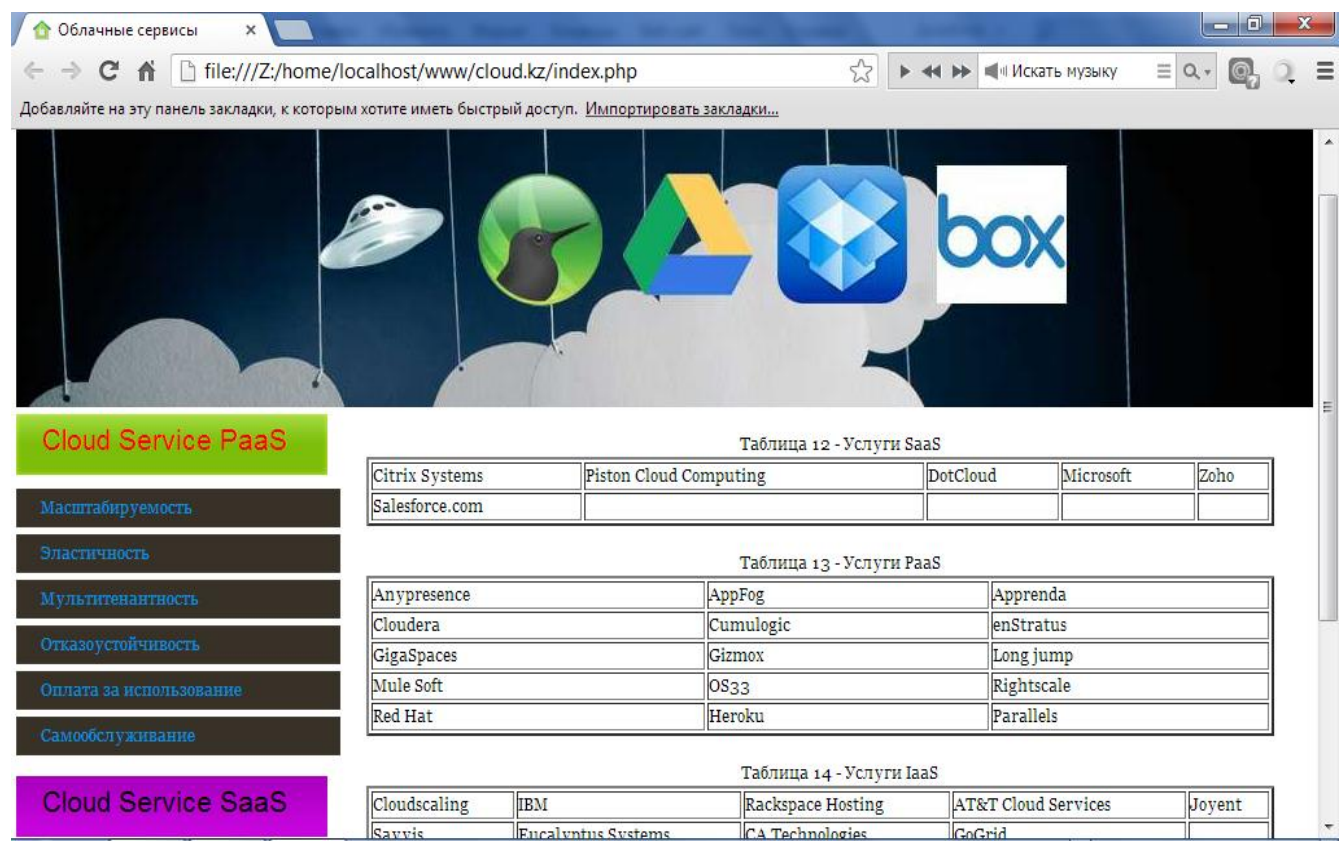

Fig.8 - Home web - portal

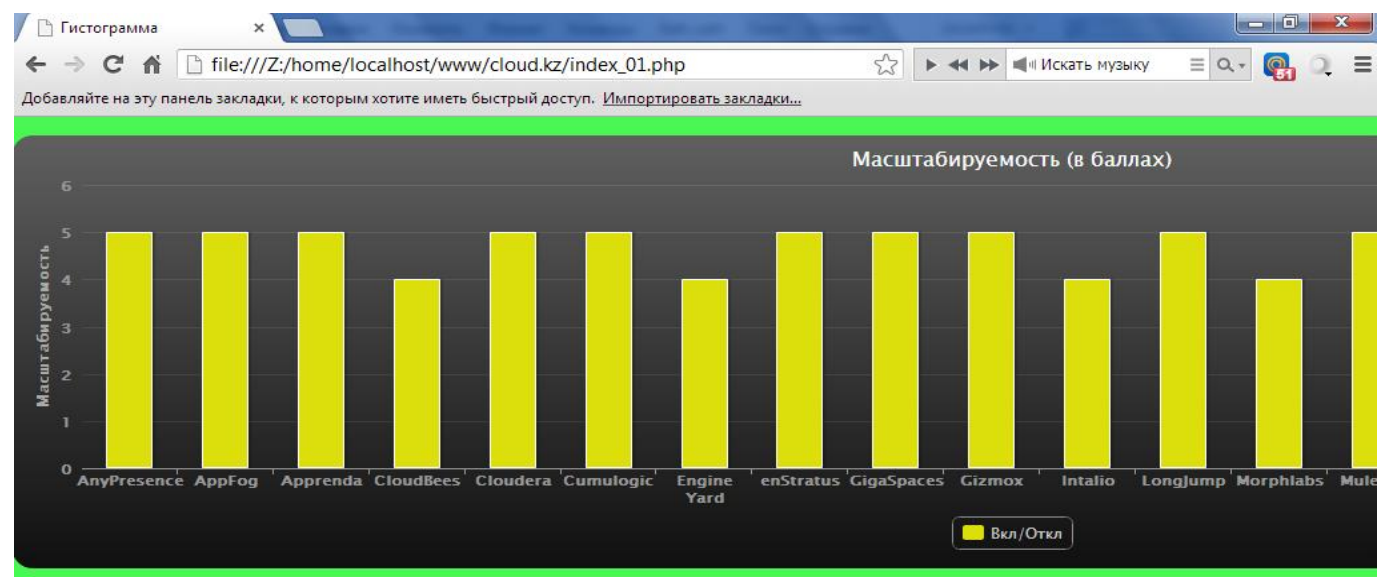

Fig.9 - Histogram of scalability. 


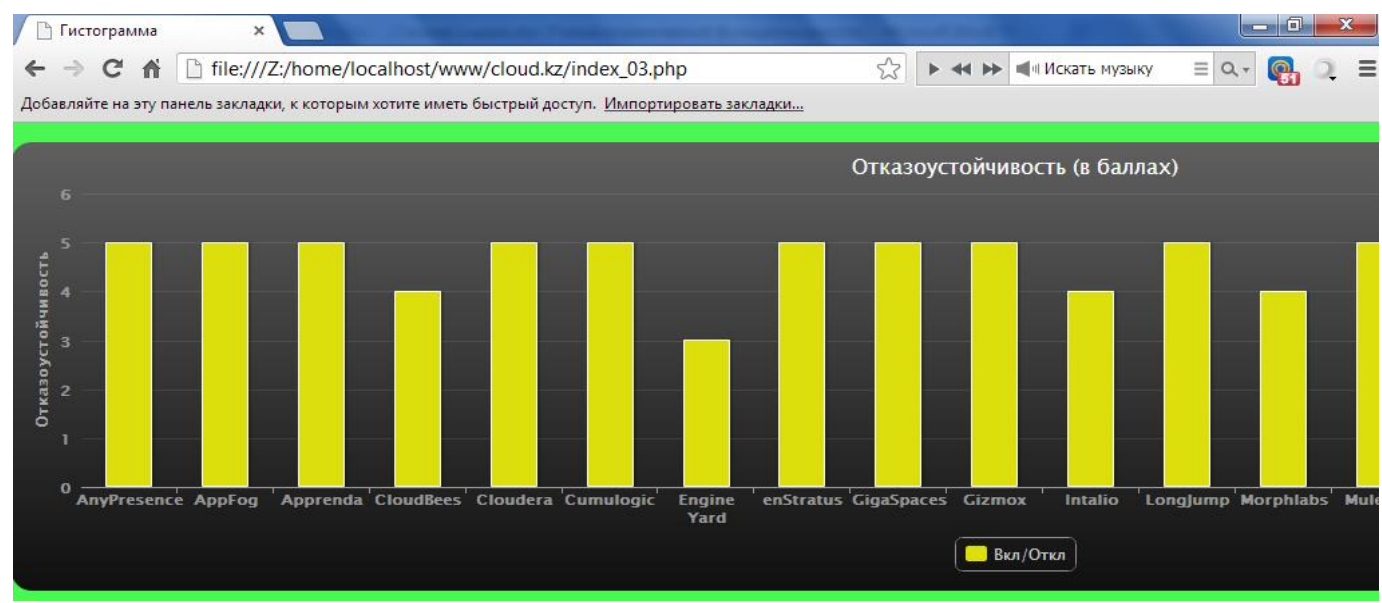

Fig.10 - Histogram of fault tolerance.

In the research work, among the 20 best providers of cloud services in the segment such as PaaS were selected by us the most suitable for servicing state agencies of
Qazaqstan on scalability, multi-tenancy, fault tolerance; such companies: (see Table 5).

Table 5 - PaaS services

\begin{tabular}{|l|c|c|c|c|c|}
\hline & \multicolumn{5}{|c|}{ Names of companies } \\
\hline $\mathbf{1}$ & Anypresence & AppFog & Apprenda & Cloudera & Cumulogic \\
\hline $\mathbf{2}$ & enStratus & GigaSpaces & Gizmox & Long jump & Mule Soft \\
\hline $\mathbf{3}$ & OS33 & Rightscale & Red Hat & Heroku & Parallels \\
\hline
\end{tabular}

Web-portal www.cloud.kz has been developed for sorting cloud services for PaaS segment and evaluating them according to the main criteria. Brief overviews of cloud products from various companies have provided. The table of twenty best companies on cloud technology which are offered by us, as PaaS has included in the international list of Cloud100. The history of development of cloud technologies in various countries of the World has been described. The analysis and monitoring the development of cloud services in many countries of the World has carried out, including in Qazaqstan.

\section{REFERENCES}

[1] Shakerkhan K.O., Khasenova G.I., Application of technology on cloud computing. Scientific journal «Bulletin of KazNTU named after Satpayev K.I.» issue number 2 in $2014 \mathrm{y}$.

[2] Peter Fingar: «DOT. CLOUD. Cloud computing is a business platform of the XXI century», Aquamarine Book, 2011, 256 pages, ISBN: 978-5-904136-21-5.

[3] Peter Saint Gwinear: «DOT. CLOUD. Cloud computingbusiness-platform for the 21st century», Aqua book 2011, 256 p. ISBN: 978-5-904136-21-5.

[4] Rittinghouse J.W., Ransom J.F. Cloud ComputingImplementation, Management, and Security//Taylor and Francis Group, 2010, 174 p.

[5] http://compress.ru/Article.aspx?id=23954

[6] http://www.cnews.ru/news/top/2016-01-25 mirovoj_rynok_publichnyh_oblachnyh_uslug_v_desyatki
[7] Kovyazin A.: Clouds for small and medium-sized businesses//open systems. - 2010, №2 - 34-37.

[8] From the idea of the «Trinity of languages» of N.A. Nazarbayev to the poly-language education in Qazaqstan http://articlekz.com/article/5831. (Date of circulation: January 18, 2017)

[9] Navin Sabharwal, Ravi Shankar. Apache CloudStack Cloud Computing. https://www.packtpub.com/virtualization-andcloud/apache-cloudstack-cloud-computing.

[10] News site: http://habrahabr.ru

[11] Tarnawsky, G.A.: first International Congress on «cloud» technology-Cloud Computing Congress SSA2010//information technology-2010-N 10.-. 77-78.

[12] Informational-analytical magazine: http://internetno.net

[13] About approval of the state program of education and science development in the Republic of Qazaqstan for 2016-2019 (Decree of the President of the Republic of Qazaqstan from March 1, 2016 № 205). http://adilet.zan.kz/kaz/docs/U1600000205. (Entrance time 16.01.2017)

[14] International it -Edition http://www.pcweek.ua

[15] George Reese. Cloud computing. http://www.ozon.ru/context/detail/id/28301660/

[16] Navin Sabharwal, Ravi Shankar. Apache CloudStack Cloud https://www.packtpub.com/virtualization-andcloud/apache-cloudstack-cloud-computing.

[17] Amit Malik. Building Clouds with Windows Azure Pack. https://www.packtpub.com/virtualization-andcloud/building-clouds-windows-azure-pack.

[18] Tarnawsky, G.A. Cloud computing/Internet/telecommunications, 2011 №2, p. 1620 . 
[19] Gillam, Lee: Cloud Computing: Principles, Systems and Applications/Nick Antonopoulos, Lee Gillam-l.: Springer, 2010.-379 p. - (Computer Communications and Networks) — ISBN 9781849962407.

[20] Mell Peter and Grance Timothy. The NIST Definition of Cloud Computing. Recommendations of the National Institute of Standards and Technology. NIST (20 October 2011).

\section{Authors' Profiles}

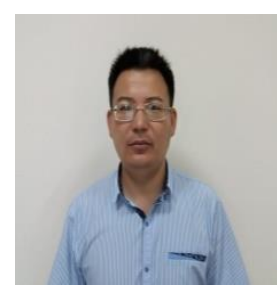

TSc Kapan Oralgazyolu Shakerkhan ${ }^{1}$ Shakarim State University of Semey city/Informatics \& Information Technologies Department, Semey, 071409, Qazaqstan, kapanshakerkhan@mail.ru

Associated Prof. Ermek Tolegenovich Abilmazhinov ${ }^{2}$, Shakarim State University of Semey city/Informatics \& Information Technologies Department, Semey, 071412, Qazaqstan, eras@mail.ru
Associated Prof. Gulsara Ezhenkhanovna Berikkhanova ${ }^{3}$, Shakarim State University of Semey city/Informatics \& Information Technologies Department, Semey, 071400, Qazaqstan, bgulsara@mail.ru

MNSc Aijan Sailauqankyzy Ryszhanova ${ }^{4}$, Shakarim State University of Semey city/Informatics \& Information Technologies Department, Semey, Qazaqstan,ras_73@mail.ru

MNSc Shynar Zhumagalievna Bolsynbekova ${ }^{5}$, Shakarim State University of Semey city/Informatics \& Information Technologies Department, Semey, Qazaqstan, bolsynbekova@mail.ru

1. Master of technical science ${ }^{1}$, Shakarim State University of Semey city, Qazaqstan

2. CTSc, associated professor $^{2}$, Shakarim State University of Semey city, Qazaqstan

3. PhD Doctor ${ }^{3}$, Shakarim State University of Semey city, Qazaqstan

4. Master of natural science ${ }^{4}$, Shakarim State University of Semey city, Qazaqstan

5. Master of natural science ${ }^{5}$, Shakarim State University of Semey city, Qazaqstan

How to cite this paper: Kapan Oralgazyolu Shakerkhan, Ermek Tolegenovich Abilmazhinov, " Development of a Method for Choosing Cloud Computing on the Platform of Paas for Servicing the State Agencies", International Journal of Modern Education and Computer Science(IJMECS), Vol.11, No.9, pp. 14-25, 2019.DOI: 10.5815/ijmecs.2019.09.02 